\title{
Avaliação do consumo alimentar e associação com o diagnóstico clínico e fatores de risco em pacientes cardiopatas
}

\author{
Evaluation of food consumption and association with clinical diagnosis and risk factors in \\ heart patients
}

\section{Evaluación del consumo y asociación de alimentos con diagnóstico clínico y factores de riesgo en pacientes con enfermedades del corazón}

Alissa Raquel Amoras Moura1*, Rodrigo Tavares Nogueira1, Fábio Costa de Vasconcelos², Paloma Barbosa da Silva ${ }^{3}$, Cybelle Barbosa Pires da Silva ${ }^{3}$, Ana Cândida de Souza Barroso², Jamile Cristine Marques Barros ${ }^{1}$.

\section{RESUMO}

Objetivo: Avaliar o consumo alimentar e associá-lo ao diagnóstico clínico e fatores de risco de pacientes cardiopatas. Métodos: Estudo do tipo descritivo, transversal e inferencial, desenvolvido com 32 indivíduos adultos e idosos, de 23 a 88 anos, em um hospital público de Belém do Pará. Aplicou-se um questionário sobre fatores de riscos relacionados às doenças cardiovasculares e outro de frequência alimentar do tipo MEDFICTS. Resultados: Verificou-se uma predominância do sexo masculino (84,38\%) e idade entre 49 a 61 anos (37,50\%). Diagnosticou-se mais frequentemente a Insuficiência cardíaca congestiva (ICC) associada ao infarto agudo do miocárdio (IAM). Observou-se que os principais fatores de risco foram: tabagismo, etilismo, alto consumo de carne vermelha, baixo consumo de frutas e estresse antes do diagnóstico. Cerca de $37,5 \%$ dos pacientes precisavam fazer alguma mudança dietética, sendo tal alteração associada ao diagnóstico de IAM/ICC (91.46) e aterosclerose (84.46). O alto consumo de carne vermelha também prevaleceu em pacientes com diagnóstico de IAM-ICC $(89,47)$. Conclusão: Identificou-se um percentual considerável de inadequação do consumo alimentar entre os participantes, sendo tal ingestão associada ao diagnóstico de IAM, ICC e aterosclerose, assim como a prevalência de um alto consumo de carne vermelha em pacientes com diagnóstico de IAM-ICC.

Palavras-chave: Consumo de alimentos, Cardiopatias, Inquéritos nutricionais.

\begin{abstract}
Objective: Assess food consumption and associate it with the clinical diagnosis and risk factors of patients with heart disease. Methods: Descriptive, cross-sectional and inferential study, developed with 32 adult and elderly individuals, aged 23 to 88 years, in a public hospital in Belém do Pará. A questionnaire was applied on risk factors related to cardiovascular diseases and another of food frequency. of type MEDFICTS. Results: There was a predominance of males (84.38\%) and age between 49 and 61 years (37.50\%). Congestive heart failure (CHF) associated with acute myocardial infarction (AMI) was most frequently diagnosed. It was observed that the main risk factors were: smoking, alcohol consumption, high consumption of red meat, low consumption of fruits and stress before diagnosis. Approximately $37.5 \%$ of patients needed to make some dietary change, and this change was associated with the diagnosis of AMI / CHF (91.46) and atherosclerosis (84.46). The high consumption of red meat also prevailed in patients diagnosed with CHF-AMI (89.47). Conclusion: A considerable percentage of inadequate food consumption was identified among the participants, such intake being associated with the diagnosis of $\mathrm{AMI}$, CHF and atherosclerosis, as well as the prevalence of a high consumption of red meat in patients diagnosed with CHF-AMI.
\end{abstract}

Keywords: Food consumption, Heart diseases, Nutrition surveys.

\section{RESUMEN}

Objetivo: Valorar el consumo de alimentos y asociarlo al diagnóstico clínico y factores de riesgo de los pacientes con cardiopatía. Métodos: Estudio descriptivo, transversal e inferencial, desarrollado con 32 adultos y ancianos, de 23 a 88 años, en un hospital público de Belém do Pará. Se aplicó un cuestionario sobre factores de riesgo relacionados con enfermedades cardiovasculares y otro de frecuencia alimentaria de tipo MEDFICTS. Resultados: Predominó el sexo masculino (84,38\%) y la edad entre 49 y 61 años (37,50\%). La insuficiencia cardíaca congestiva (ICC) asociada a infarto agudo de miocardio (IAM) fue el más diagnosticado. Se observó que los principales factores de riesgo fueron: tabaquismo, consumo de alcohol, alto consumo de carnes rojas, bajo consumo de frutas y estrés antes del diagnóstico.

\footnotetext{
1 Universidade do Estado do Pará (UEPA), Belém - PA. *E-mail: alissa_amoras@hotmail.com

${ }^{2}$ Centro Universitário do Pará (CESUPA), Belém - PA.

3 Universidade da Amazônia (UNAMA), Belém - PA.
} 
Aproximadamente el $37,5 \%$ de los pacientes necesitaron realizar algún cambio en la dieta, y este cambio se asoció con el diagnóstico de IAM / ICC $(91,46)$ y aterosclerosis $(84,46)$. El alto consumo de carnes rojas también predominó en pacientes diagnosticados de IAM-ICC $(89,47)$. Conclusión: Se identificó un porcentaje considerable de consumo inadecuado de alimentos entre los participantes, estando asociada dicha ingesta al diagnóstico de IAM, ICC y aterosclerosis, así como a la prevalencia de un alto consumo de carne roja en pacientes diagnosticados de ICC-IAM.

Palabras clave: Consumo de alimentos, Cardiopatías, Encuestas nutricionales.

\section{INTRODUÇÃO}

As doenças cardiovasculares (DCV) são consideradas uma das principais causas de mortalidade mundial, representando 17,9 milhões de óbitos em 2016 no Brasil. Neste mesmo ano, as DCV foram responsáveis por $28 \%$ das mortes dentre as doenças crônicas não transmissíveis (DCNT) (WHO, 2018). Dados apresentados pela Sociedade Brasileira de Cardiologia estimam um aumento de óbitos no decorrer dos anos, atingindo 383.961 pessoas neste país, em 2017 (SBC, 2015).

As projeções para 2030 apontam que as patologias do aparelho circulatório afetarão $73 \%$ do total de mortalidade no mundo, além de serem os motivos mais expressivos de incapacidade e limitação da sociedade (ALVES CG e MORAIS NETO OL, 2015; TESTON EF, et al., 2016). Tais condições associam-se a custos com tratamento e internações hospitalares, além de outras despesas provenientes da diminuição da produtividade, ausência do trabalho e os impactos sobre a qualidade de vida dos indivíduos doentes e sua família (SIQUEIRA ASE, et al., 2017; WHO, 2011).

Vale ressaltar que os Fatores de Risco (FR) aumentam a probabilidade de desencadeamento da DCV e identificá-los pode ajudar a encontrar ameaças emergentes à saúde dos indivíduos, oportunizando a sua prevenção (PRÉCOMA DB, et al., 2019). São considerados alguns dos FR para a doença cardíaca: alimentação inadequada, dislipidemia, obesidade, tabagismo, etilismo, inatividade física e estresse (BONOTTO GM, et al., 2016; MEDEIROS FILHO RA, et al., 2018; PRÉCOMA DB, et al., 2019). Estes fatores podem ser modificados com intervenções que visem mudanças do estilo de vida e de hábitos não saudáveis (BONOTTO GM, et al., 2016; MEDEIROS FILHO RA, et al., 2018).

Entende-se que o processo fisiopatológico mais difundido para o surgimento das DCV é a origem de desordens metabólicas, como a Resistência à Insulina (RI), bem como a predisposição genética, que acaba corroborando para o desenvolvimento da RI e a superabundância de Ácidos Graxos Livres (AGL) na corrente sanguínea, em decorrência do excesso de peso. É importante destacar, também, o papel dos adipócitos na obesidade, pois estes aumentam de volume devido ao maior aporte de triglicerídeos, ocorrendo a liberação de AGL para a corrente sanguínea e sua posterior deposição como estoque ectópico de gordura nas células musculares, cardíacas, hepáticas e células-beta pancreáticas (GUYTON AC; HALL JE, 2006; LUSIS AJ; ATTIE AD; REUE K, 2008).

No fígado, o acúmulo de AGL permite a gliconeogênese e o aumento da produção de triglicerídeos e lipoproteínas de muito baixa densidade (VLDL - Very Low Density Lipoprotein). Esse excesso gera VLDL remanescentes, denominado de LDL (Low Density Lipoprotein). Dessa forma, quando há excesso de LDL na corrente sanguínea, a aterosclerose é favorecida pela distribuição de colesterol para os tecidos extrahepáticos, resultando na formação de placas de ateroma e consequentemente, risco de doenças cardiovasculares (GUYTON AC; HALL JE, 2006).

Segundo a $7^{a}$ Diretriz Brasileira de Hipertensão Arterial, o sucesso para o tratamento com medidas nutricionais depende da adoção de um plano alimentar saudável e sustentável. No meio científico, a dieta DASH (Dietary Approaches to Stop Hypertension) é considerada um dos tratamentos nutricionais primordiais para o controle da pressão. Esta enfatiza o consumo de frutas, hortaliças e laticínios com baixo teor de gordura, inclui a ingestão de cereais integrais, frango, peixe, frutas, oleaginosas e preconiza a redução da ingestão de carne vermelha, doces e bebidas com açúcar. Outra dieta com efeito hipotensor é a do Mediterrâneo, que contém frutas, hortaliças e cerais integrais, porém possui quantidades generosas de azeite de oliva, além da ingestão moderada de vinho (MALACHIAS MVB, et al., 2017). 
As dietas vegetarianas preconizam o consumo de alimentos de origem vegetal, como frutas, hortaliças, grãos e leguminosas e excluem ou raramente incluem carnes. Essas dietas são relacionadas com valores mais baixos de pressão arterial (PA). Com relação ao consumo de sódio, os resultados ainda são controversos, pois, enquanto alguns sustentam que o consumo muito baixo poderia elevar o risco de DCV, contudo, outros sustentam que a diminuição de sódio reduz o risco cardiovascular (MALACHIAS MVB, et al., 2017). De acordo com Organização Mundial da Saúde (OMS), os indivíduos adultos consomem menos que 5 gramas $(\mathrm{g})$ de sal por dia, o equivalente a 2.000 miligramas $(\mathrm{mg})$ de sódio por dia. Contudo, tem-se observado que a população brasileira ingere uma média de $11,4 \mathrm{~g} / \mathrm{dia}$, corroborando com as altas prevalências de DCV encontradas (BEZERRA VM, 2019).

As preocupações atuais a respeito da alimentação, devido ao alto consumo de alimentos com elevado teor de açúcar, sódio e lipídeos são pertinentes, pois, principalmente a qualidade da gordura na dieta pode influenciar significativamente a saúde da população (WHO, 2015). De todas as gorduras ingeridas, a saturada tem grande relação com alterações no perfil lipídico, como o aumento das concentrações de colesterol total e das lipoproteínas de baixa densidade. Com isso, pode-se considerar este nutriente como aterogênico, e consequentemente como um risco para o sistema circulatório (ROS E, et al., 2015).

Dessa forma, o consumo alimentar é uma variável complexa, pois, dependendo de como é realizado, pode colaborar com o aparecimento de doenças ou atuar como um fator de proteção contra elas. Já é bem estabelecido, por exemplo, que o tipo e a quantidade de gordura interferem sobre os fatores de risco cardiovascular (BRUNORI EHFR, et al., 2015). Diante do exposto, esta pesquisa tem como objetivo avaliar o consumo alimentar e relacionar com diagnóstico clínico e fatores de risco associados às doenças cardiovasculares de pacientes internados em um hospital público de Belém do Pará.

\section{MÉTODOS}

Conduziu-se uma pesquisa do tipo descritiva, transversal e inferencial. Para realizá-la foi utilizada uma amostra contendo 32 indivíduos internados nas clínicas de cardiologia e de atendimento e triagem, em um hospital de referência nesta especialidade, no estado do Pará. A coleta de dados teve duração de 60 dias, sendo esta uma média de atendimentos no período, de pacientes com doença cardiovascular.

Incluíram-se nesta pesquisa os indivíduos acometidos por doença cardiovascular, de ambos os sexos, com faixa etária $\geq 20$ anos, os quais estiveram internados no referido hospital no período da coleta de dados e que assinaram o Termo de Consentimento Livre e Esclarecido (TCLE). Quanto aos critérios de exclusão, não puderam fazer parte do estudo os pacientes sem diagnostico de doença cardiovascular, com idade inferior a 20 anos e que se negaram a assinar o TCLE.

A obtenção dos dados foi executada por meio de ficha pré-estabelecida, contendo as seguintes variáveis: número do prontuário, sexo, idade, diagnóstico e questionário sobre fatores de riscos relacionados às doenças cardiovasculares. Também foi aplicado o Questionário de Frequência Alimentar (QFA) por meio ferramenta Meat, Eggs, Dairy, Fried food, in baked goods, convenience, foods, Table foods, Snack (MEDFICTS).

O questionário utilizado (MEDFICTS) para a pesquisa foi extraído do programa Nacional Cholesterol Education Program (NCEP) adotado pela American Heart Association, projetado especificamente para avaliar a adesão dos pacientes às dietas, com objetivo principal de reduzir, gradativamente, o risco de doença coronariana através da redução de colesterol LDL dietético. As etapas 1 e 2 de dietas do programa concentram-se em uma redução de gordura total para $30 \%$ ou menos de energia diária, com redução progressiva de gordura saturada e colesterol (KRIS-ETHERTON P, et al., 2001; TAYLOR AJ, et al., 2003).

Logo após este passo, os pontos foram totalizados na coluna de resultados, cujos mesmos foram somados em cada lado do QFA e interpretados de acordo com a tabela de classificação pré-existente. Caso o total ocorresse de forma maior ou igual a 70, indicar-se-ia uma necessidade de alterações na rotina alimentar, ou seja, que houvesse uma redução na ingestão de gordura total, saturada e de colesterol. Se porventura os valores obtidos estivessem entre 40 a 70, este resultado indicaria que o entrevistado está mantendo ou seguindo uma dieta saudável e se o total fosse menor que 40, sugerir-se-ia um não consumo excessivo de gorduras (KRIS-ETHERTON P, et al., 2001). 
O questionário é dividido da seguinte forma: a coluna apresenta categorias com itens de gêneros alimentícios distribuídos ao grupo 1 (desejável) ou ao grupo 2 (indesejável), levando em consideração o teor de gordura dos alimentos. Os valores numéricos são atribuídos a cada grupo de alimentos com pesos baseados no consumo semanal e no tamanho da porção. Logo, é pontuado usando o total da quantidade de ingestão ajustada pela qualidade, resultando em uma possível faixa de pontuação de 0 a 216 pontos. As pontuações mais baixas indicam dietas contendo menos gorduras dietéticas. A literatura de validação afirma que a ferramenta pode ser auto administrada de 3 a 5 minutos e avaliada pelo pesquisador e/ou profissional da saúde em aproximadamente 2 minutos (TAYLOR AJ, et al., 2003).

É uma ferramenta disponível para a avaliação do risco cardiovascular, entretanto, o questionário não apresenta validação para a população brasileira, porém, os objetivos do mesmo concentram-se em consumir $<7 \%$ de gordura saturada, $<200 \mathrm{mg}$ de colesterol diário e $<30 \%$ de energia de gordura total, o que colabora com o que é preconizado pela Diretrizes Brasileiras sobre Dislipidemias e Prevenção da Aterosclerose (FALUDI AA, et al., 2017). Dessa forma, sua aplicação faz-se interessante para observar o risco cardiovascular em diversas populações, dentre elas, a brasileira.

O trabalho foi executado de acordo com as Normas de Pesquisa Envolvendo Seres Humanos do Conselho Nacional de Saúde e com aprovação do Comitê de ética em Pesquisa com o CAEE: 24713013.2.0000.5173 e parecer 537.020, autorização da instituição do hospital, além da assinatura pelos pacientes do Termo de Consentimento Livre e Esclarecido (TCLE). As tabelas e gráficos foram construídos no Microsoft Excel® 2010. Por meio da estatística descritiva (média e desvio padrão), verificou-se as variáveis quantitativas, através do BioEstat 5.3. Quanto às variáveis qualitativas, realizou-se a análise de correspondência, por meio do software STATISTICA versão 8.

\section{RESULTADOS}

A população avaliada foi composta de 32 pacientes com diagnóstico de doenças cardiovasculares, com média de idade $57,12 \pm 15,80$ anos. Nota-se uma maior proporção de indivíduos do sexo masculino em comparação ao feminino, associado a predominância do diagnóstico de insuficiência cardíaca congestiva e infarto agudo do miocárdio (Tabela 1).

Tabela 1 - Número e percentual de pacientes com doenças cardiovasculares, de acordo com a faixa etária, gênero e diagnóstico, em um hospital de referência, Belém - PA.

\begin{tabular}{ccc}
\hline Faixa Etária & $\mathbf{N}$ & $\%$ \\
\hline $23 \vdash 36$ & 04 & 12,50 \\
$36 \vdash 49$ & 04 & 12,50 \\
$49 \vdash 62$ & 12 & 37,50 \\
$62 \vdash 75$ & 08 & 25,00 \\
$75 \vdash 88$ & 04 & 12,50 \\
Total & 32 & 100,00 \\
\hline Gênero & $\mathbf{N}$ & $\%$ \\
\hline Feminino & 05 & 15,63 \\
Masculino & 27 & 84,38 \\
Total & 32 & 100,00 \\
\hline Diagnóstico Clínico & $\mathbf{N}$ & $\%$ \\
\hline ICC - IAM & 25 & 78,13 \\
Outros & 07 & 21,87 \\
Total & 32 & 100,00 \\
\hline
\end{tabular}

Fonte: Moura ARA, et al., 2020.

Observa-se que os fatores de risco: tabagismo, etilismo, alto consumo de carne vermelha, baixo consumo de frutas e estresse antes do diagnóstico, foram estatisticamente significantes, mostrando dependência entre as variáveis e diagnóstico (Tabela 2). 
Tabela 2 - Prevalência de fatores de risco dos pacientes com doenças cardiovasculares, em um hospital de referência, Belém - PA.

\begin{tabular}{|c|c|c|c|c|}
\hline \multirow{2}{*}{ Fatores de Risco } & Não & \multirow{2}{*}{$\begin{array}{c}\text { Sim } \\
\text { n (\%) }\end{array}$} & \multirow{2}{*}{$\mathbf{X}^{2}$} & \multirow{2}{*}{ p- valor } \\
\hline & n $\quad(\%)$ & & & \\
\hline Tabagismo & $10(31,25)$ & $22(68,75)$ & 4,50 & $<0,05^{\star}$ \\
\hline Etilismo & $08(25,00)$ & $24(75,00)$ & 8,00 & $<0,05^{\star}$ \\
\hline Antecedentes Familiares & $14(43,75)$ & $18(56,25)$ & 0,50 & 0,47 \\
\hline Sedentarismo & $13(40,62)$ & $19(59,38)$ & 1,12 & 0,28 \\
\hline Hipertensão Arterial Sistêmica & $17(53,12)$ & $15(46,88)$ & 0,12 & 0,72 \\
\hline Diabetes Tipo 2 & $21(65,62)$ & $11(34,38)$ & 3,12 & 0,07 \\
\hline Dislipidemia & $18(56,25)$ & $14(43,75)$ & 0,50 & 0,47 \\
\hline Alto consumo de carne vermelha & $03(09,38)$ & $29(90,62)$ & 21,12 & $<0,05^{*}$ \\
\hline Baixo consumo de frutas & $09(28,12)$ & $23(71,88)$ & 6,12 & $<0,05^{\star}$ \\
\hline Baixo consumo de verdura & $16(50,00)$ & $16(50,00)$ & 0,00 & 1,00 \\
\hline Estresse antes do diagnóstico & $09(28,12)$ & $23(71,88)$ & 6,12 & $<0,05^{\star}$ \\
\hline
\end{tabular}

Legenda: *p-valor: <0,05 (nível de significância). Nota: $X^{2}$ : Qui-quadrado de aderência.

Fonte: Moura ARA, et al., 2020.

Verificou-se por meio do questionário MEDFICTS que, aproximadamente, $40 \%$ precisavam fazer mudança dietética (Gráfico 1).

Gráfico 1 - Classificação qualitativa do consumo da dieta usual de pacientes com diagnóstico de doenças cardiovasculares, segundo questionário MEDFICTS, em um hospital de referência, Belém - PA.

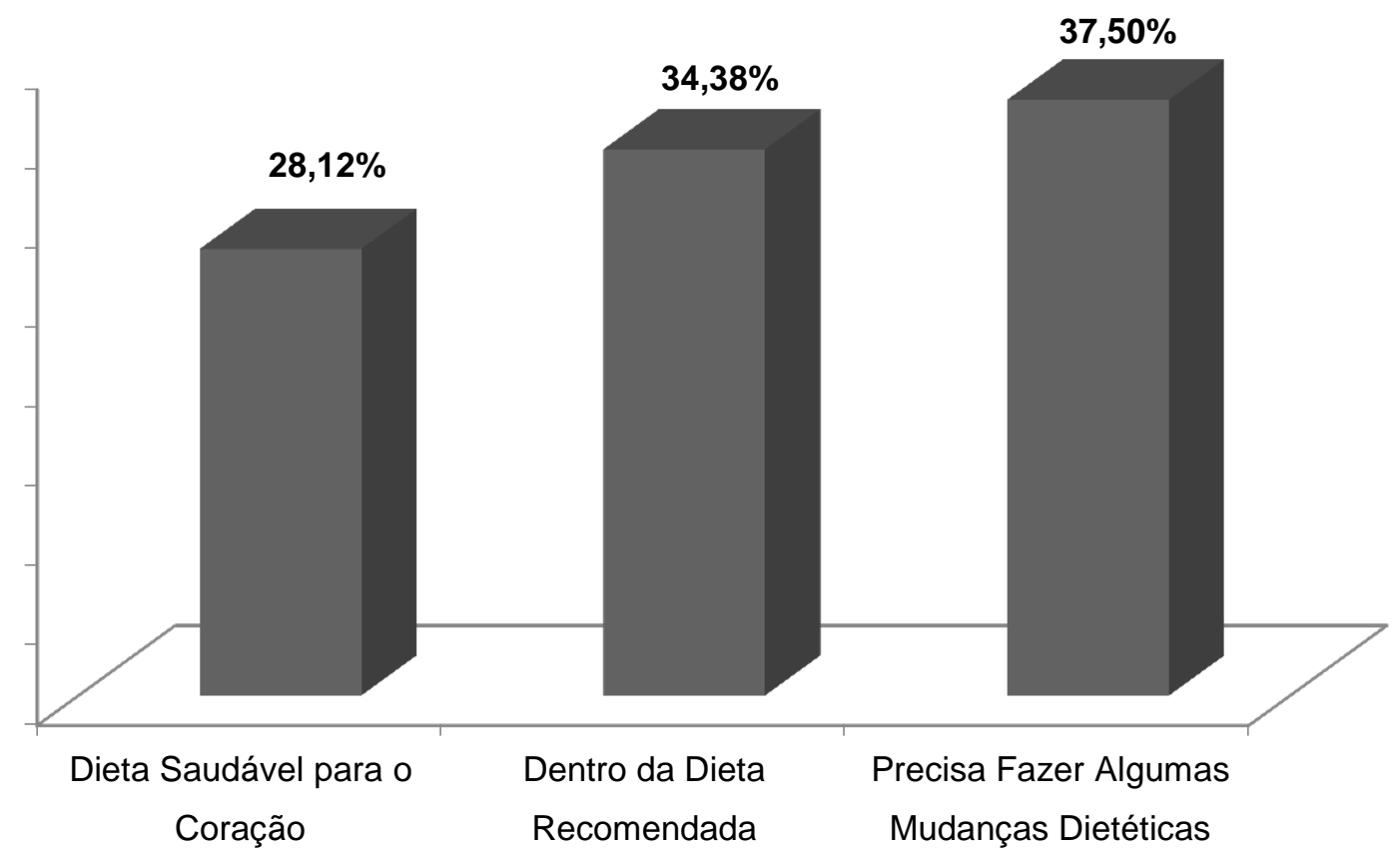

MEDFICTS

Fonte: Moura ARA, et al., 2020. 
A partir dos dados apresentados, as categorias das variáveis estudadas foram inerentes ao diagnóstico clínico e fatores de risco. Dessa forma, o valor do qui-quadrado, $p$ e critério Beta possibilitaram condições viáveis para aplicação Diagnóstico Clínico versus MEDFICTS e Diagnóstico Clínico versus Alto consumo de carne vermelha. Ressalta-se, ainda, que as variáveis testadas e consideradas independentes não foram apresentadas (Tabela 3).

Tabela 3 - Resultados do teste de dependência das variáveis: Diagnóstico Clínico e fatores de risco, de pacientes com doenças cardiovasculares em um Hospital Público de Belém - PA.

\begin{tabular}{lcccccc}
\hline Variáveis & $\mathbf{X}^{\mathbf{2}}$ & $\boldsymbol{g . I}$ & $\boldsymbol{P}$ & $\boldsymbol{L}$ & $\boldsymbol{C}$ & $\boldsymbol{B}$ \\
\hline Diagnóstico Clínico e MEDFICTS & 14,08 & 7 & 0,00 & 8 & 3 & 5,70 \\
Diagnóstico Cínico e Alto consumo de carne vermelha & 17,45 & 7 & 0,00 & 8 & 2 & 6,56 \\
\hline
\end{tabular}

Legenda: $x^{2}$ = qui-quadrado; $g . l$ = grau de liberdade; $p=$ nível descritivo; $I$ = número de linhas; $c=$ número de colunas.

Fonte: Moura ARA, et al., 2020.

De acordo com os resultados obtidos, destaca-se uma associação forte entre os pacientes que apresentaram diagnóstico de Insuficiência Cardíaca Congestiva (ICC) associada ao Infarto Agudo do Miocárdio (IAM) e aterosclerose com dieta inadequada, isto é, com alto teor de gordura e que precisa ser modificada, com alto nível de significância. Observa-se que os pacientes que tiveram diagnóstico clínico de endocardite, estenose mitral e doença cardíaca congênita apresentaram associação com dieta saudável (Tabela 4).

Tabela 4 - Resíduos e Níveis de Confiança Resultantes da Aplicação da Análise de Correspondência às Variáveis: Diagnóstico Clínico e MEDFICTS, de pacientes com doenças cardiovasculares, em um Hospital Público de Belém - PA.

\begin{tabular}{lccc}
\hline MEDFICTS & & \\
\hline Diagnóstico Clínico & $\begin{array}{c}\text { Dieta saudável para o } \\
\text { coração }\end{array}$ & $\begin{array}{c}\text { Dentro da dieta } \\
\text { recomendada }\end{array}$ & $\begin{array}{c}\text { Precisa fazer } \\
\text { algumas mudanças }\end{array}$ \\
\hline \multirow{2}{*}{ Endocardite } & 1.40 & -0.59 & -0.61 \\
& $\mathbf{8 3 . 7 1}$ & 0.00 & 0.00 \\
\hline \multirow{2}{*}{ Estenose Mitral } & 1.36 & -0.59 & -0.61 \\
& $\mathbf{8 2 . 4 7}$ & 0.00 & 0.00 \\
\hline \multirow{2}{*}{ ICC-IAM } & -0.39 & 0.14 & 1.72 \\
\multirow{2}{*}{ Dissecção da Aorta } & 0.00 & 11.02 & $\mathbf{9 1 . 4 6}$ \\
\hline \multirow{2}{*}{ Doença Cardíaca Congênita } & -0.53 & -0.59 & 1.02 \\
\hline \multirow{2}{*}{ Arritmia Cardíaca } & 0.00 & 0.00 & 69.26 \\
\hline \multirow{2}{*}{ Estenose Aórtica } & 1.38 & -0.59 & -0.61 \\
& 83.10 & 0.00 & 0.00 \\
\hline \multirow{2}{*}{ Aterosclerose } & -0.53 & 1.02 & -0.61 \\
& 0.00 & 69.19 & 0.00 \\
\hline
\end{tabular}

Fonte: Moura ARA, et al., 2020.

Destaca-se uma associação forte entre os pacientes que apresentaram diagnóstico de Insuficiência Cardíaca Congestiva (ICC) associada ao Infarto Agudo do Miocárdio (IAM) e consumo elevado de carne vermelha com significância de 89,471\% (Tabela 5). 
Tabela 5 - Resíduos e Níveis de Confiança Resultantes da Aplicação da Análise de Correspondência às Variáveis: Diagnóstico Clínico e Alto consumo de carne vermelha, de pacientes com doenças cardiovasculares, em um Hospital Público de Belém - PA.

\begin{tabular}{lcc}
\hline \multirow{2}{*}{ DIAGNÓSTICO CLÍNICO } & ALTO CONSUMO DE CARNE VERMELHA \\
& Não & Sim \\
\hline \multirow{2}{*}{ Endocardite } & 1.01 & -0.84 \\
& 68.85 & 0.00 \\
\hline \multirow{2}{*}{ Estenose Mitral } & 1.02 & -0.87 \\
& 69.32 & 0.00 \\
\hline \multirow{2}{*}{ ICC-IAM } & -0.90 & 1.62 \\
& 0.00 & 89.47 \\
\multirow{2}{*}{ Dissecção da Aorta } & 0.50 & -0.87 \\
& 38.29 & 0.00 \\
\multirow{2}{*}{ Doença Cardíaca Congênita } & -0.50 & 0.29 \\
\multirow{2}{*}{ Arritmia Cardíaca } & 0.00 & 22.72 \\
\hline \multirow{2}{*}{ Estenose Aórtica } & -0.50 & 0.29 \\
& 0.00 & 22.72 \\
\hline \multirow{2}{*}{ Aterosclerose } & 0.45 & -0.87 \\
& 34.73 & 0.00 \\
\hline
\end{tabular}

Fonte: Moura ARA, et al., 2020.

\section{DISCUSSÃO}

A presença de doenças cardiovasculares tem aumentado nos últimos anos, como resultado da maior adesão ao estilo de vida ocidental, caracterizado pelo consumo de alimentos de baixo valor nutricional, aumento do sedentarismo, redução de atividade física e/ou lazeres (DUMITH SC, et al., 2011).

Segundo Kessler EL et al. (2019), as doenças cardiovasculares podem ser influenciadas pelos níveis hormonais entre os gêneros, pois, embora as mulheres sofram frequentes oscilações hormonais durante a vida, acredita-se que tais efeitos podem retardar o aparecimento de DCV em mulheres pós menopausa, podendo ser uma das justificativas para o predomínio de doenças cardiovasculares em homens, tal como mostra a Tabela 1.

Tal condição também pode ser explicada por informações contidas em estudos como no de Vigilância de Fatores de Risco e Proteção para Doenças Crônicas por Inquérito Telefônico (VIGITEL), observando-se uma maior prevalência de hábitos e fatores como tabagismo, sobrepeso, consumo elevado de bebidas e sedentarismo nos homens em comparação com as mulheres (MUSSI FC e TEIXEIRA JRB, 2018).

A principal condição cardiológica que acometeu os participantes foi o IAM, com quase $90 \%$ de ocorrência. A partir do estudo de carga global de doenças realizado entre 1990 e 2015, observou-se que a patologia cardiovascular que mais se apresentou em brasileiros durante esse período foi a cardiopatia isquêmica (KHAN SS, et al., 2017).

A prevalência de cardiopatias é explicada devido à transição epidemiológica ocorrida nas últimas décadas, com diminuição de doenças infecciosas, respiratórias e parasitárias, ocorrendo, entretanto, um aumento de Doenças Crônicas não Transmissíveis (DCNT), como neoplasias e doenças cardiorrespiratórias (ERVATTI LR, et al., 2015).

Os principais fatores de risco para complicações cardíacas apresentados pelos participantes do estudo foram o alto consumo de carne vermelha, baixo consumo de frutas, tabagismo, etilismo e estresse, todos com nível de significância estatística, quando comparados com os que não apresentaram tais alterações. 
De acordo com a Atualização da Diretriz de Prevenção Cardiovascular da Sociedade Brasileira de Cardiologia (2019), existem alguns fatores de risco cardiovasculares clássicos e também outros considerados de risco emergente. Entre os fatores, estão: espiritualidade, fatores socioeconômicos e ambientais, dislipidemias, obesidade, tabagismo, antecedentes familiares, hipertensão arterial e alcoolismo (PRÉCOMA DB, et al., 2019).

Em adultos saudáveis, o consumo de baixas a moderadas quantidades de álcool geralmente não apresenta impacto de curto prazo substancial na hemodinâmica ou na pressão arterial (PA). Contudo, dados sugerem que o consumo excessivo de álcool, ou seja, mais que cinco drinques em uma única sessão estão associados a aumentos transitórios na PA que variam de 4 a $7 \mathrm{mmHg}$ para sistólica e de 4 a $6 \mathrm{mmHg}$ para diastólica (PIANO MR, 2017).

É importante ressaltar, também, que a maioria das pessoas é exposta a traumas psicológicos ou eventos estressores que geram acúmulo de vivências, podendo levar a um risco aumentado de doenças, como a morbidade cardiovascular (SONG H, et al., 2018). O efeito fisiológico de um agente estressor agudo pode afetar diretamente o sistema circulatório que, em condições favoráveis, inicia eventos cardiovasculares agudos. Um estudo recente com 4.178 indivíduos entre 30 a 70 anos indica associação entre transtorno do estresse pós traumático e a doença cardiovascular (SCHERRER JF, et al., 2019).

Outro fator que notavelmente causa alterações pressóricas é o tabagismo, este é prevalente em todo mundo e seu efeito na DVC, bem como na mortalidade por todas as causas, está bem estabelecido (MUEZZINLER A, et al., 2015). Kunutsor SK, et al. (2018) avaliou a exposição ao tabagismo com o risco de DCV de 3.455 indivíduos, afirmando que os fumantes graves apresentaram risco aumentado para DCV, independente dos fatores de risco cardiovasculares.

Entende-se que o ato de fumar induz a peroxidação lipídica e o metabolismo do óxido nítrico, bem como a elevação do estresse oxidativo celular e, consequentemente, o processo inflamatório e a disfunção endotelial e plaquetária. Relacionando com a influência no âmbito nutricional, a nicotina promove redução do apetite e maior saciedade devido à sua ação sobre o hormônio leptina e o neuropeptídeo $\mathrm{Y}$, assim, desempenhando um importante papel na ingestão alimentar que promove a diminuição do peso corporal (JACONDINO CB, et al., 2019).

A partir do QFA MEDFICTS foi possível avaliar a qualidade da alimentação dos pacientes antes da internação, e classificar de acordo com a pontuação obtida (KRIS-ETHERTON P, et al., 2001). O resultado mostrou que a maioria dos pacientes precisava fazer algumas mudanças dietéticas, pois a alimentação encontrava-se inadequada, fato que pode ter contribuído para o surgimento da patologia (ANAND SS, et al., 2015).

Da amostra, $31,25 \%$ dos pacientes classificaram-se dentro da dieta recomendada e, apenas $25 \%$ em dieta saudável para o coração. A maioria dos pacientes que estava se alimentando de forma imprópria era composta por homens adultos. Observa-se, também, que neste estudo os pacientes com diagnóstico clínico de endocardite, estenose mitral e doença cardíaca congênita apresentaram associação com "dieta saudável para o coração", com significância estatística de 83,71\%; 82,47\% e 83,10\%, respectivamente.

É possível encontrar nas bases de dados pesquisas envolvendo a aplicabilidade e validação da ferramenta MEDFICTS em outras populações, com desfechos similares ao encontrado neste artigo, descrevendo uma boa correlação com o consumo de gorduras total, saturada, colesterol e com a aderência à dieta (MOCHARI H, et al., 2008). Conduto, usando outras ferramentas para avaliação do consumo alimentar, pesquisas relatam ingestão alimentar de colesterol acima das diretrizes recomendadas em 92\% de pacientes portadores da síndrome coronariana aguda, apresentando consumo elevado de sódio e lipídios em 46,7\% e 32,7\%, respectivamente, na amostra estudada (BRUNORI EHFR, et al., 2015).

A carne vermelha apresenta várias substâncias em sua composição que quando consumidos de maneira excessiva e errônea, podem causar prejuízos à saúde. Dentre os principais podem-se destacar os compostos mutagênicos que são as aminas heterocíclicas (HCAs) e hidrocarbonetos aromáticos policíclicos (PAHs), que são gerados através do cozimento em altas temperaturas $\left(>150^{\circ} \mathrm{C}\right)$ e por longos 
períodos; os poluentes ambientais que são considerados as toxinas inorgânicas; o alto teor de gordura saturada; o alto teor de sal em carnes processadas e a produção de trimetilamina - $N$ - óxido (TMAO) pelo microbioma intestinal (ALISSON-SILVA F, et al., 2016).

Um estudo realizado pela Associação Americana de Pessoas Aposentadas encontrou associação entre o consumo de carne vermelha e processada com a mortalidade pela DCV por todas as causas (ETEMADI $A$ et al, 2017). No entanto, um estudo realizado em adultos coreanos de meia idade mostrou que 0 consumo de carne não processada apresentou associação inversa com a DCV (PARK K, et al., 2017). Corroborando com a pesquisa de Mertens E, et al. (2017) que relata que o padrão alimentar caracterizado por pão branco, manteiga, banha, bebidas adoçadas e baixo consumo de grãos integrais foi relacionado a um maior risco de desenvolver DCV e acidente vascular cerebral (AVC).

Para o melhor entendimento sobre alimentação e saúde cardiovascular, existe disponível na literatura uma iniciativa brasileira voltada a orientações dietéticas para cardiopatas, chamada de dieta cardioprotetora, a qual reúne informações sobre o consumo de açúcares, teor de gordura, colesterol, ácidos graxos saturados, sódio e densidade calórica, de forma a identificá-los por cores, buscando alertar quanto aos alimentos com maiores e menores teores dos nutrientes citados anteriormente, buscando modificar e melhorar o consumo alimentar dos portadores de cardiopatias (WEBER B, et al., 2019).

O referido estudo foi conduzido em vários centros de referência em cardiologia no Brasil, com pacientes que se enquadravam e se voluntariaram para participar, o qual obteve resultados positivos quanto à adesão de uma dieta considerada mais saudável (WEBER B, et al., 2019). Quanto ao consumo de carnes vermelhas e doenças cardiovasculares, a literatura sugere que uma ingestão adequada dessa fonte de proteína não se mostra associada a desfechos cardíacos (MICHA R, et al., 2013; BRONZATO S e DURANTE A, 2017).

Em um recente estudo com indivíduos americanos, os autores concluíram que um maior consumo de carne vermelha, assim como o de carne processada e de aves mostrou-se associado a maiores chances de risco cardiovascular. $\mathrm{O}$ consumo de carnes vermelhas e de carne processada associou-se ainda a um leve aumento no risco de mortalidade por todas as causas (ZHONG VW, et al., 2020). Entende-se que uma avalição rápida e precisa da ingestão de gordura na dieta é extremamente importante na tomada de decisão clínica em relação ao aconselhamento dietético para a redução do risco cardiovascular.

Os métodos de avalição do consumo alimentar existentes e consolidados para a população brasileira podem ser aplicados, também, para investigar o risco cardíaco, bem como outras doenças. Porém, atualmente não existe uma ferramenta com o objetivo direcionado para 0 rastreio cardiovascular para 0 público em questão, dessa forma, faz-se necessária a validação do questionário aplicado em outros estudos de maiores proporções para esses indivíduos.

\section{CONCLUSÃO}

A partir da avaliação do conteúdo observado pela ferramenta MEDFICTS, identificou-se que quase $40 \%$ dos participantes ingeriam uma alimentação considerada inadequada para sua condição clínica. Tal consumo se associou com maior predominância aos diagnosticados com infarto agudo do miocárdio, juntamente à insuficiência cardíaca congestiva e aterosclerose. Assim como o alto consumo de carne vermelha, que se apresentou como um dos principais fatores de risco para cardiopatias, também prevaleceu em pacientes com diagnóstico de IAM-ICC.

\section{REFERÊNCIAS}

1. ALVES CG, MORAIS NETO OL. Tendência da mortalidade prematura por doenças crônicas não transmissíveis nas unidades federadas brasileiras. Ciência Saúde Coletiva, 2015; 20(3): 641-654.

2. ALISSON-SILVA F, et al. Human risk of disease associated with red meat intake: analysis of current theories and proposed role for metabolic incorporation of a non-human sialic acid. Mol Aspects Med, 2016; 51: 16-30.

3. ANAND SS, et al. Food Consumption and its impact on Cardiovascular Disease: Importance of Solutions focused on the globalized food system: A report from the workshop convened by the World Heart Federation. J Am Coll Cardiol, 2015; 66(14): 1590-1614. 
4. BEZERRA VM. Técnica dietética em preparações especiais: teoria e prática de laboratório. Rio de Janeiro: Rubio, 2019; $320 \mathrm{p}$.

5. BONOTTO GM, et al. Conhecimento dos fatores de risco modificáveis para doença cardiovascular entre mulheres e seus fatores associados: um estudo de base populacional. Ciência Saúde Coletiva, 2016; 21(1): 293-302.

6. BRONZATO S, DURANTE A. A contemporary review of the relationship between red meat consumption and cardiovascular risk. Int J Prev Med, 2017; 8(1): 1-7.

7. BRUNORI EHFR, et al. Ingestão alimentar e estresse em pacientes com síndrome coronariana aguda. Rev. Bras. Enferm, 2015; 68(5): 810-816.

8. DUMITH SC, et al. Worldwide prevalence of physical inactivity and its association with human development index in 76 countries. Prev Med, 2011; 53(1-2):24-28.

9. ERVATTI LR, et al. Mudança demográfica no brasil no início do século XX: subsídios para as projeções da população. Instituto Brasileiro de Geografia e Estatística 2015. Disponível em: https://biblioteca.ibge.gov.br/visualizacao/livros/liv93322.pdf. Acesso em: 02 mai. 2020.

10. ETERNADI A, et al. Mortality from different causes associated with meat, heme iron, nitrates and nitrites in the NIH-AARP Diet and health Study: population based cohort study. BMJ, 2017; 357:1957.

11. FALUDI AA, et al. Atualização da diretriz brasileira de dislipidemias e prevenção da aterosclerose - 2017. Arquivos Brasileiros de Cardiologia [Internet], 2017;109(1).

12. GUYTON AC, HALL JE. Tratado de Fisiologia Médica. 11 ed. Rio de Janeiro: Guanabara Koogan, cap. 10, p.747-758, 2006.

13. JACONDINO CB, et al. Associação do tabagismo com biomarcadores REDOX e fatores de risco cardiometabólicos em idosos. Saúde Coletiva, 2019; 27(1): 45-52.

14. KHAN SS, et al. Molecular and physiological manifestations and measurement of aging in humans. Aging Cell, 2017; 16(4): 624-633.

15. KESSLER EL, et al. Sex-specific influence on cardiac structural remodeling and therapy in cardiovascular disease. Bio Sex Differ, 2019; 10:7.

16. KUNUTSOR SK, et al. Self-reported smoking, urine cotinine, and risk of cardiovascular disease: findings from the PREVEND (Prevention of renal and vascular end-stage disease) prospective cohort study. J Am Heart Assoc, 2018; 7(10): $1-15$.

17. KRIS-ETHERTON $P$, et al. Validation for MEDFICTS, a dietary assessment instrument for evaluating adherence to total and saturated fat recommendations of the National Cholesterol Education Program Step 1 and Step 2 diets. J Am Diet Assoc, 2001; 101: 81-86.

18. LUSIS AJ, ATTIE AD, REUE K. Metabolic syndrome: from epidemiology to systems biology. Nature Reviews Genetics, v.9, n.11, p. 819-830, 2008.

19. MALACHIAS MVB, et al. 7ª Diretriz Brasileira de Hipertensão Arterial. Brazilian Journal of Hypertension, $2017 ; 24$ (1): 1-90.

20. MEDEIROS FILHO RA, et al. Prevalência de comportamentos e fatores de risco para doenças cardiovasculares em população de hipertensos no norte de Minas Gerais, Brasil. Rev Fund Care Online, 2018; 10(1): 90-6.

21. MERTENS E, et al. Dietary patterns in relation to cardiovascular disease incidence and risk markers in a middle-aged british male population: data from the caerphilly prospective study. Nutrients, 2017; 91(1): 75.

22. MICHA R, et al. Unprocessed red and processed meats and risk of coronary artery disease and type 2 diabetes - An updated review of the evidence. Curr Atheroscler Rep, 2013; 14(6): 515-524.

23. MOCHARI H, et al. Validation of the MEDFICTS Dietary Assessment Questionnaire in a Diverse Population. J Am Diet Assoc, 2008; 108(5): 817-822.

24. MUEZZINLER A, et al. Smoking and all-cause mortality in older adults: results from the chances consortium. Am J Prev Med, vol. 49(5):e53-e63, 2015.

25. MUSSI FC, TEIXEIRA JRB. Doenças isquêmicas do coração e masculinidade como fatores de risco cardiovascular. Rev Cubana Enferm, 2018; 34(2).

26. PARK K, et al. Unprocessed meat consumption and incident cardiovascular diseases in Korean Adults: The Korean genome and epidemiology study (KoGES). Nutrients, 2017; 95(5): 498.

27. PRÉCOMA DB, et al. Atualização da Diretriz de Prevenção Cardiovascular da Sociedade Brasileira de Cardiologia - 2019. Arq Bras Cardiol, 2019; 113(4): 1-105.

28. PIANO MR. Alcohol's effects on the cardiovascular system. Alcohol Res, 2017; 38(2): 219-241.

29. ROS E, et al. Consenso sobre las grasas y aceites en la alimentación de la población española adulta; postura de la Federación Española de Sociedades de Alimentación, Nutrición y Dietética (FESNAD). Nutr Hosp, 2015; 32(2): $435-477$.

30. SIQUEIRA ASE, et al. Análise do impacto econômico das doenças cardiovasculares nos últimos cinco anos no Brasil. Arq Bras Cardiol, 2017; 109(1): 39-46.

31. SCHERRER JF, et al. Comorbid conditions explain the association between posttraumatic stress disorder and incident cardiovascular disease. J Am Heart Assoc. 2019; 8(4): e011133.

32. SOCIEDADE BRASILEIRA DE CARDIOLOGIA (SBC). Cardiômetro: mortes por doenças cardiovasculares no Brasil [Internet] 2015. Disponível em: http://www.cardiometro.com.br/anteriores.asp. Acesso em: 20 jul. 2020.

33. SONG H, et al. Association of stress-related disorders with subsequent autoimmune disease. JAMA, vol. 319(23):2388$2400,2018$.

34. TAYLOR AJ, et al. Validation of the MEDFICTS dietary questionnaire: a clinical tool to assess adherence to American Heart Association dietary fat intake guidelines. Nutrition Journal, 2003; 2(4).

35. TESTON EF, et al. Fatores associados às doenças cardiovasculares em adultos. Medicina, 2016; 49(2): 95-102.

36. WEBER B, et al. Implementation of a Brazilian Cardioprotective Nutritional (BALANCE) Program for improvement on quality of diet and secondary prevention of cardiovascular events: A randomized, multicenter trial. Am Heart J, 2019; 215: $187-197$.

37. WHO. WORLD HEALTH ORGANIZATION. 2011. The Global Economic Burden of Non-communicable Diseases - Reducing the Economic Impact of Non Communicable Diseases in Low- and Middle-Income Countries. Geneva: World Economic Forum.

38. WHO. WORLD HEALTH ORGANIZATION. 2018. In: Noncommunicable diseases country profiles. Geneva: World Health Organization. Disponível em: https://www.who.int/nmh/publications/ncd-profiles-2018/en/. Acesso em: 20 jul. 2020.

39. WHO. WORLD HEALTH ORGANIZATION. 2015. In: Noncommunicable diseases progress monitor. Geneva: World Health Organization. Disponível em: https://www.who.int/nmh/publications/ncd-progress-monitor-2015/en/. Acesso em: 03 mai. 2020.

40. ZHONG VW, et al. Associations of processed meat, unprocessed red meat, poultry, or fish intake with incident cardiovascular disease and all-cause mortality. JAMA Intern Med, 2020; 180(4): 503-512. 